\title{
Key traveller groups of relevance to spatial malaria transmission: a survey of movement patterns in four sub-Saharan African countries
}

John M. Marshall ${ }^{1,2 *}$, Mahamoudou Touré ${ }^{3}$, André Lin Ouédraogo ${ }^{4,5}$, Micky Ndhlovu', Samson S. Kiware Ashley Rezai ${ }^{1}$, Emmy Nkhama ${ }^{6}$, Jamie T. Griffin ${ }^{1}$, T. Deirdre Hollingsworth ${ }^{1,8}$, Seydou Doumbia ${ }^{3}$, Nicodem J. Govella ${ }^{7}$, Neil M. Ferguson ${ }^{1}$ and Azra C. Ghani ${ }^{1}$

\begin{abstract}
Background: As malaria prevalence declines in many parts of the world due to widescale control efforts and as drug-resistant parasites begin to emerge, a quantitative understanding of human movement is becoming increasingly relevant to malaria control. However, despite its importance, significant knowledge gaps remain regarding human movement, particularly in sub-Saharan Africa.
\end{abstract}

Methods: A quantitative survey of human movement patterns was conducted in four countries in sub-Saharan Africa: Mali, Burkina Faso, Zambia, and Tanzania, with three to five survey locations chosen in each country. Questions were included on demographic and trip details, malaria risk behaviour, children accompanying travellers, and mobile phone usage to enable phone signal data to be better correlated with movement. A total of 4352 individuals were interviewed and 6411 trips recorded.

Results: A cluster analysis of trips highlighted two distinct traveller groups of relevance to malaria transmission: women travelling with children (in all four countries) and youth workers (in Mali). Women travelling with children were more likely to travel to areas of relatively high malaria prevalence in Mali $(\mathrm{OR}=4.46,95 \% \mathrm{Cl}=3.42-5.83)$, Burkina Faso ( $\mathrm{OR}=1.58,95 \% \mathrm{Cl}=1.23-1.58)$, Zambia $(\mathrm{OR}=1.50,95 \% \mathrm{Cl}=1.20-1.89)$, and Tanzania $(\mathrm{OR}=2.28$, $95 \% \mathrm{Cl}=1.71-3.05)$ compared to other travellers. They were also more likely to own bed nets in Burkina Faso $(\mathrm{OR}=1.77,95 \% \mathrm{Cl}=1.25-2.53)$ and Zambia $(\mathrm{OR}=1.74,95 \% \mathrm{Cl}=1.342 .27)$, and less likely to own a mobile phone in Mali $(\mathrm{OR}=0.50,95 \% \mathrm{Cl}=0.39-0.65)$, Burkina Faso $(\mathrm{OR}=0.39,95 \% \mathrm{Cl}=0.30-0.52)$, and Zambia $(\mathrm{OR}=0.60$, $95 \% \mathrm{Cl}=0.47-0.76)$. Malian youth workers were more likely to travel to areas of relatively high malaria prevalence $(\mathrm{OR}=23,95 \% \mathrm{Cl}=17-31)$ and for longer durations (mean of 70 days cf 21 days, $\mathrm{p}<0.001)$ compared to other travellers.

Conclusions: Women travelling with children were a remarkably consistent traveller group across all four countries surveyed. They are expected to contribute greatly towards spatial malaria transmission because the children they travel with tend to have high parasite prevalence. Youth workers were a significant traveller group in Mali and are expected to contribute greatly to spatial malaria transmission because their movements correlate with seasonal rains and hence peak mosquito densities. Interventions aimed at interrupting spatial transmission of parasites should consider these traveller groups.

\footnotetext{
*Correspondence: john.marshall@berkeley.edu

${ }^{2}$ Divisions of Biostatistics and Epidemiology, School of Public Health,

University of California, Berkeley, CA, USA

Full list of author information is available at the end of the article
} 
Keywords: Plasmodium falciparum, Spatial transmission, Cluster analysis, Women with children, Youth workers, Mobile phones, Mali, Burkina Faso, Zambia, Tanzania

\section{Background}

As a vector-borne disease, malaria is spread alternately between its human and mosquito hosts. Humans travel much larger distances than mosquitoes and consequently human movement plays a dominant role in parasite dispersal [1, 2]. Significant funding is currently being invested in global malaria control [3] and as transmission declines [3, 4], a quantitative understanding of human movement is important to determine how best to target interventions [2]. Quantifying the dispersal of malaria parasites is particularly relevant once overall transmission has declined because, in combination with knowledge of environmental heterogeneity, it allows sources and sinks of transmission to be identified. Control programmes can then be designed that target the 'hot spots' and 'hot pops' of transmission [5-7]. Human movement is also of relevance to the spread of drug-resistant malaria parasites, which have recently emerged in Southeast Asia [8].

Despite the importance of human movement data in planning disease control, significant data gaps remain [9], particularly in sub-Saharan Africa, where over $90 \%$ of malaria-related deaths occur [3]. One new data source that is increasingly being used as a proxy for human movement is anonymous mobile phone signal data [2, $10,11]$. This is a powerful and comprehensive data source that can conceivably be obtained at large scale. However, one drawback is that it may give a biased estimate of movement patterns in many African countries where affluent men are more likely to be mobile phone owners [12], phone sharing is common among rural women, and many individuals use multiple SIM cards due to nonoverlapping provider coverage [13]. An additional limitation is that young children rarely have mobile phones, but are the demographic among which malaria is most prevalent. Data on child movement are also often lacking in household surveys [14], where the number of trips away in the last year is measured without information on children accompanying family members.

Substantial qualitative work has been conducted describing the types of human movements in Africa, many of which are relevant to malaria transmission. For example, in the Sahel, a semi-arid region beneath the Sahara Desert including parts of Mali and Burkina Faso, seasonal rains are correlated with both agricultural labour movements and malaria transmission due to an abundance of vector-breeding sites [5, 15]. Rural youths tend to leave their villages after a season's harvest to look for casual work in nearby cities, stay away for several months and then return to help with farm work in the next agricultural season [16-18]. There is also a strong ethnic dimension to movements-for instance, the Songhai in Mali are known as good traders, setting up shops in cities throughout the country and migrating seasonally to sell goods [19]. In East and Southern Africa, there is a strong culture of sending young children to boarding school [20], which is a source of youth circulation. Across the continent, migration to urban areas is becoming increasingly attractive for all ethnic groups as a source of employment, education and permanent settlement [21, 22], which itself leads to increased short-term visits from other family members [23].

To gain a better quantitative understanding of these patterns, a human movement survey was conducted in four countries with ongoing malaria transmission: two in West Africa (Mali and Burkina Faso), one in East Africa (Tanzania) and one in Southern Africa (Zambia) (Fig. 1). In consultation with local researchers, three to five survey locations were chosen in each country that were expected to capture a wide range of traveller groups. Survey respondents were asked about trips for which they had spent at least one night away from home, since the main African malaria vectors, Anopheles gambiae and Anopheles funestus, bite at night. Questions were also asked about demography and trip details to gain a better descriptive understanding of movement, about mobile phone usage to enable phone signal data to be better correlated with self-reported movement patterns, and about malaria risk behaviour and children accompanying travellers, since children are the most common parasite carriers.

\section{Methods \\ Study sites}

The survey was undertaken in four sub-Saharan African countries with ongoing malaria transmission-Mali, Burkina Faso, Zambia, and Tanzania (Fig. 1). In Mali and Burkina Faso, seasonal agricultural labour movements are common [15]. These countries are representative of a number of Sahelian countries in West Africa, including Senegal, Niger and Benin. These populations are highly dependent on arable land, with $80 \%$ of the Malian labour force involved in fishing or agricultural activities [24] and $90 \%$ of Burkinabe labour force involved in agriculture [24]. To explore the impact of survey timing on results, the Mali survey was conducted during consecutive rainy 


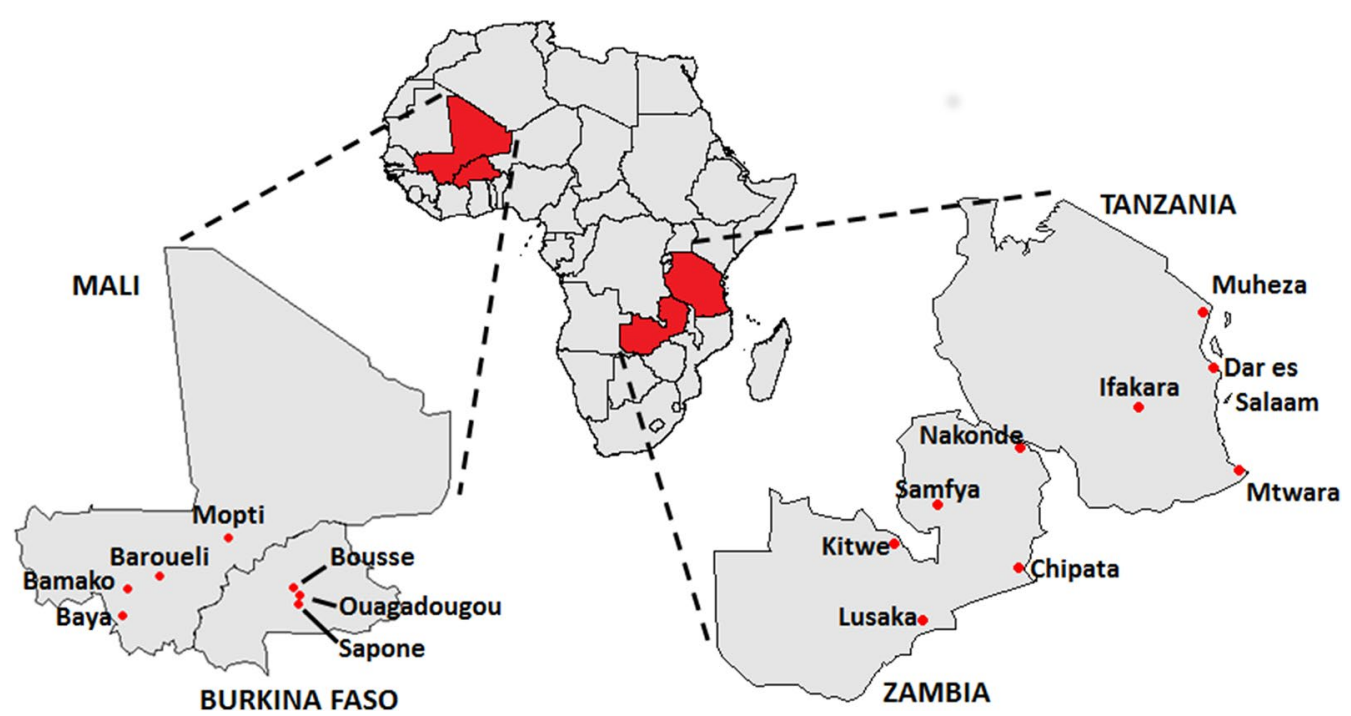

Fig. 1 Survey locations in Mali, Burkina Faso, Zambia and Tanzania

and dry seasons. Budgetary constraints did not permit this for other countries. Zambia and Tanzania both have a comprehensive national transport network, allowing their populations to be mobile on a large scale [24]. The population of both countries is also highly dependent on arable land; however, here agriculture is more stable yearround, leading to less seasonal movements [24].

The survey sites were chosen in collaboration with local malaria researchers and medical anthropologists. These were sites that: (a) were judged to capture a wide range of traveller groups present in the country; and, (b) had an existing relationship with the local researchers. Sampling of households within each study site was randomized, however, the study sites themselves were a judgement/ convenience sample. Hence, inferences based on these results reflect the collection of study sites rather than the countries at large.

The Mali survey was conducted in Bamako, the capital city and largest urban centre, two fishing villages in Baya, two farming villages in Baroueli, and in Mopti and Fatoma, a commercial centre and village, respectively, approximately $460 \mathrm{~km}$ northeast of Bamako. The Burkina Faso survey was conducted in Ouagadougou, the capital and largest city, and two nearby locations-Sapone, an agricultural village approximately $50 \mathrm{~km}$ south of Ouagadougou, and Boussé, a major centre of agriculture and trade approximately $50 \mathrm{~km}$ north of Ouagadougou. The Zambia survey was conducted in five locations-Lusaka, the capital and largest city; Samfya, a central fishing town; Kitwe, an urban trading town in the Copperbelt; Nakonde, a town in the northeast bordering Tanzania; and, Chipata, a rural town in the east bordering Malawi. The Tanzania survey was conducted in four locations-Dar es Salaam, the capital and largest city; Ifakara, a small rural town on the edge of the Kilombero valley; Muheza, a small rural town near the Indian Ocean and the border with Kenya; and, Mtwara, an agricultural town with a growing mining industry near the Indian Ocean and Mozambique.

\section{Survey questions}

Participants were recruited within each location using random sampling techniques (Additional file 1) and EpiCollect 2.0 as a data collection interface [25]. Participants were eligible for inclusion if they were 16 years of age or older and reported making at least one overnight trip in the last year. This parallels a question asked in the nationally representative Demographic and Health surveys (DHS) [14]. This allowed the survey to include a higher overall number of respondents providing information on travel patterns. The DHS survey data may be used to obtain an overall estimate of the frequency of travel and the characteristics of non-travellers.

Participants were asked a series of questions about short- and long-term circular movements over the last year (purpose, duration, month of departure, number of accompanying children on each trip), basic demographic information (age, gender, number of children under the age of five), mobile phone usage (ownership, reception, frequency of carriage, usage of a mobile phone), and malaria (perceived risk of malaria, bed net ownership, usage). Questions were also asked about migratory patterns. Details are provided in Additional file 1. The questionnaire was prepared in English, but administered in the languages of the local population. 
A trip was considered short-term if its duration was 2 weeks or less, and long-term if its duration was longer than 2 weeks but less than a year. Details were recorded for the three most recent short- and long-term trips. Travel within the ward, commune or city of origin was not included. Destinations were recorded at the level of commune or ward via dropdown lists in Epicollect. Dropdown lists were populated using shape files for each of the study countries, allowing locations to be geocoded. Study participants were interviewed in Mali during the rainy season of September/ October 2010 and the dry season of March 2011, in Burkina Faso during the rainy season of July 2011, in Zambia during the cool dry season of July/August 2012, and in Tanzania during the long rainy season of March 2013.

\section{Ethics}

Ethical approval for the study was granted by the Imperial College Research Ethics Committee, UK and the Institutional Review Boards of the Malaria Research and Training Center in Mali, the Centre National de Recherche et de Formation sur le Paludisme in Burkina Faso, ERES Converge in Zambia and the Ifakara Health Institute Research Ethics Board in Tanzania. The survey was anonymous and informed consent was obtained from all participants.

\section{Statistical analysis}

A descriptive analysis of the demographic data, trip properties, mobile phone usage, behaviour, and malariarelated variables was undertaken using individual trips as the unit of analysis. Hierarchical cluster analysis was performed using trip properties (logarithm of distance travelled, trip duration, season of departure, purpose, whether children accompanied, whether the origin and destination were rural or urban) and the demographics of the travellers (age, gender, number of children under 5 years, number of trips in the last year) as input variables in the FactoMineR package in $\mathrm{R}$ [26]. All recorded trips were included in the analysis. On average, 1.47 trips were analysed per person; however within-person correlation was not accounted for. As both continuous and categorical variables were analysed, the continuous variables were scaled to unit variance and the categorical variables were transformed into a disjunctive data table and scaled using multiple correspondence analysis. Missing values were replaced by the mean of the respective variable. The cluster analysis was performed both on the overall dataset and separately by country. Clusters were retained if they explained a significant amount of the variance in the combined analysis, determined if an additional cluster was associated with an inertia gain of $Q>0.5$ [26].

Multivariable logistic regression was used to identify differences in mobile phone usage behaviour and malaria-related variables between the identified trip clusters. Malaria-related variables included bed net ownership, bed net usage at the origin and destination of travel, perceived malaria risk, and estimated malaria prevalence at the origin and destination of travel. Perceived malaria risk was derived from the following survey questions"Do you think you are at risk of getting malaria where you live?" and "Do you perceive there to be a risk of malaria where you travelled to?" with high, medium and low response options. Estimated malaria prevalence was based on spatially stratified 2010 estimates of parasite prevalence in 2-10 years old [27] aggregated to the ward/ commune level or to the next highest administrative level at which the origin and destination were resolved. Mobile phone-related variables included phone ownership, which was included in the full logistic regression model, and the frequency of carrying and using a phone, travelling with the phone, reception issues and phone sharing behaviour, which were analysed as part of a secondary logistic regression model conditional upon phone ownership.

\section{Results \\ Survey results}

In total, 4352 individuals were interviewed-1588 from Mali, 721 from Burkina Faso, 1093 from Zambia and 950 from Tanzania (Additional file 2). Table 1 summarizes the demographics of those surveyed. These reflect the random sample of people who made at least one overnight trip in the last year from the judgement/ convenience sample of survey sites in each country. The Mali sample had significantly more male interviewees (854 males $c f 730$ females, $\mathrm{p}=0.002$ ), while the Zambia sample had significantly more female interviewees (671 females $c f 413$ males, $\mathrm{p}<0.001$ ). Interviewees in the Tanzanian sample reported more children under the age of five residing in the same residence than in the other countries (mean of 1.56 children $c f 0.85$ for other countries, $\mathrm{p}<0.001$ ) and interviewees in the Zambia sample reported more children under the age of five than in the Mali and Burkina Faso samples (mean of 1.00 children $c f$ 0.79 for Mali/Burkina Faso, $\mathrm{p}<0.001$ ). Interviewees in the Burkina Faso and Zambia samples reported significantly more trips than interviewees in the Mali and Tanzania samples (mean of 2.75 trips $c f 1.50$ trips, $\mathrm{p}<0.001$ ), while DHS surveys reported a higher proportion of the populations of Zambia and Tanzania as having travelled in the last year compared to the populations of Mali and Burkina Faso [14, 28-30]. Bed net ownership was significantly lower among interviewees in the Zambian sample compared to the Tanzanian and Burkinabe sample (71\% ownership cf $86 \%$ for Tanzania/Burkina Faso, $\mathrm{p}<0.001)$. Mobile phone ownership was significantly lower among 
Table 1 Descriptive statistics of interviewees in each national sample

\begin{tabular}{|c|c|c|c|c|c|c|c|c|}
\hline & \multicolumn{2}{|l|}{ Mali } & \multicolumn{2}{|c|}{ Burkina Faso } & \multicolumn{2}{|c|}{ Zambia } & \multicolumn{2}{|c|}{ Tanzania } \\
\hline & $N$ & $\%(\mathrm{Cl})$ & $N$ & $\%(\mathrm{Cl})$ & $N$ & $\%(\mathrm{Cl})$ & $N$ & $\%(\mathrm{Cl})$ \\
\hline Total & 1588 & & 721 & & 1093 & & 950 & \\
\hline \multicolumn{9}{|l|}{ Gender } \\
\hline Female & 730 & $46(43-48)$ & 349 & $48(45-52)$ & 671 & $61(58-64)$ & 473 & $50(47-53)$ \\
\hline Male & 854 & $54(51-56)$ & 365 & $51(47-54)$ & 413 & $38(35-41)$ & 470 & $49(46-53)$ \\
\hline \multicolumn{9}{|l|}{ Age } \\
\hline $16-29$ & 760 & $48(45-50)$ & 316 & $44(40-48)$ & 482 & $44(41-47)$ & 416 & $44(41-47)$ \\
\hline $30-45$ & 511 & $32(30-35)$ & 284 & $39(36-43)$ & 395 & $36(33-39)$ & 381 & $40(37-43)$ \\
\hline$>45$ & 317 & $20(18-22)$ & 118 & $16(14-19)$ & 206 & $19(17-21)$ & 147 & $16(13-18)$ \\
\hline Mean number children <5 years (range) & & $0.81(0-9)$ & & $0.77(0-5)$ & & $1.01(0-7)$ & & $1.56(0-5)$ \\
\hline Mean number trips (range) & & $1.56(1-51)$ & & $3.42(1-100)$ & & $2.30(1-100)$ & & $1.40(1-20)$ \\
\hline Proportion who travelled (DHS) & & 0.313 & & 0.291 & & 0.431 & & 0.427 \\
\hline \multicolumn{9}{|l|}{ Own bed net } \\
\hline Yes & $\mathrm{N} / \mathrm{A}$ & $\mathrm{N} / \mathrm{A}$ & 581 & $81(78-83)$ & 778 & $71(68-74)$ & 853 & $90(88-92)$ \\
\hline No & $\mathrm{N} / \mathrm{A}$ & $\mathrm{N} / \mathrm{A}$ & 136 & $19(16-22)$ & 314 & $29(26-32)$ & 91 & $10(8-12)$ \\
\hline \multicolumn{9}{|l|}{ Own mobile phone } \\
\hline Yes & 859 & $54(52-57)$ & 527 & $73(70-76)$ & 715 & $65(63-68)$ & 726 & $76(74-79)$ \\
\hline No & 727 & $46(43-48)$ & 191 & $26(23-30)$ & 330 & $30(27-33)$ & 217 & $23(20-26)$ \\
\hline
\end{tabular}

The number of individuals $(N)$ and percentage of the sample (exact binomial $95 \%$ confidence interval), or mean (range) are shown. All interviewees did not answer all questions, hence there are some missing values

interviewees in the Malian sample compared to the Burkinabe and Tanzanian sample (54\% ownership cf $75 \%$ for Tanzaznia/Burkina Faso, $\mathrm{p}<0.001)$. Differences between the rainy and dry season samples in Mali are described in Section 2.1 of Additional file 1 and in Additional file 3: Table S1.

\section{Trip clusters}

A cluster analysis identified three clusters of trips in the Mali sample and two in the Burkina Faso, Zambia and Tanzania samples based on the demographic and trip variables described in Methods. In all settings, the cluster that captured the greatest amount of variance in the data consisted predominantly of trips involving women between the ages of 16 and 45 years who travelled with children, usually for family-related reasons. This trip cluster is denoted as 'women with children' (Fig. 2) or 'women travelling with children' since the cluster is dominated by trips involving women travelling with children, although the clustering algorithm also included a smaller number of trips made by other groups. In the Mali, Burkina Faso and Zambia samples, 93-94 \% of the trips in this cluster were made by women, 85,73 and $83 \%$ of whom were travelling with children, respectively. In the Tanzania sample, the cluster was less well defined, with $82 \%$ of the trips being made by women, $56 \%$ of whom travelled with children. In all four country samples, $<1 \%$ of the trips in the remaining clusters involved travel with children. The 'women with children' trip cluster also emerged in a combined, four-country cluster analysis and captured the greatest amount of variance in the data in this analysis.

Trips in the 'women with children' cluster tended to involve shorter distances than other clusters (mean of $136 \mathrm{~km}$ cf $191 \mathrm{~km}$ for other clusters, $\mathrm{p}<0.001)$. For example, in the Mali sample, the mean trip distance for trips in the 'women with children' cluster was $120 \mathrm{~km}$, compared to $157 \mathrm{~km}$ for those in other clusters, and in the Zambia sample, the mean trip distance was $161 \mathrm{~km}$ for the 'women with children' cluster, compared to $203 \mathrm{~km}$ for other trip clusters (Fig. 3a). Travellers in the 'women with children' cluster also tended to make fewer trips than those in other clusters (mean of 2.2 trips $c f 3.3$ trips for other clusters, $\mathrm{p}<0.001$ ). For example, in the Burkina Faso sample, travellers in the 'women with children' cluster made a mean of 2.5 trips in the last year, compared to travellers in other clusters who made a mean of 5.7 trips (Fig. 2f).

In the Mali sample, the cluster of trips that captured the second highest amount of variance in the data consisted predominantly of trips made by Malian youths between the ages of 16 and 29 years travelling predominantly for work-related reasons (Fig. 2). This trip cluster is denoted as 'youth workers' (Fig. 2) since the cluster is dominated by work-related trips made by youths, although the clustering algorithm again included a smaller number of trips 

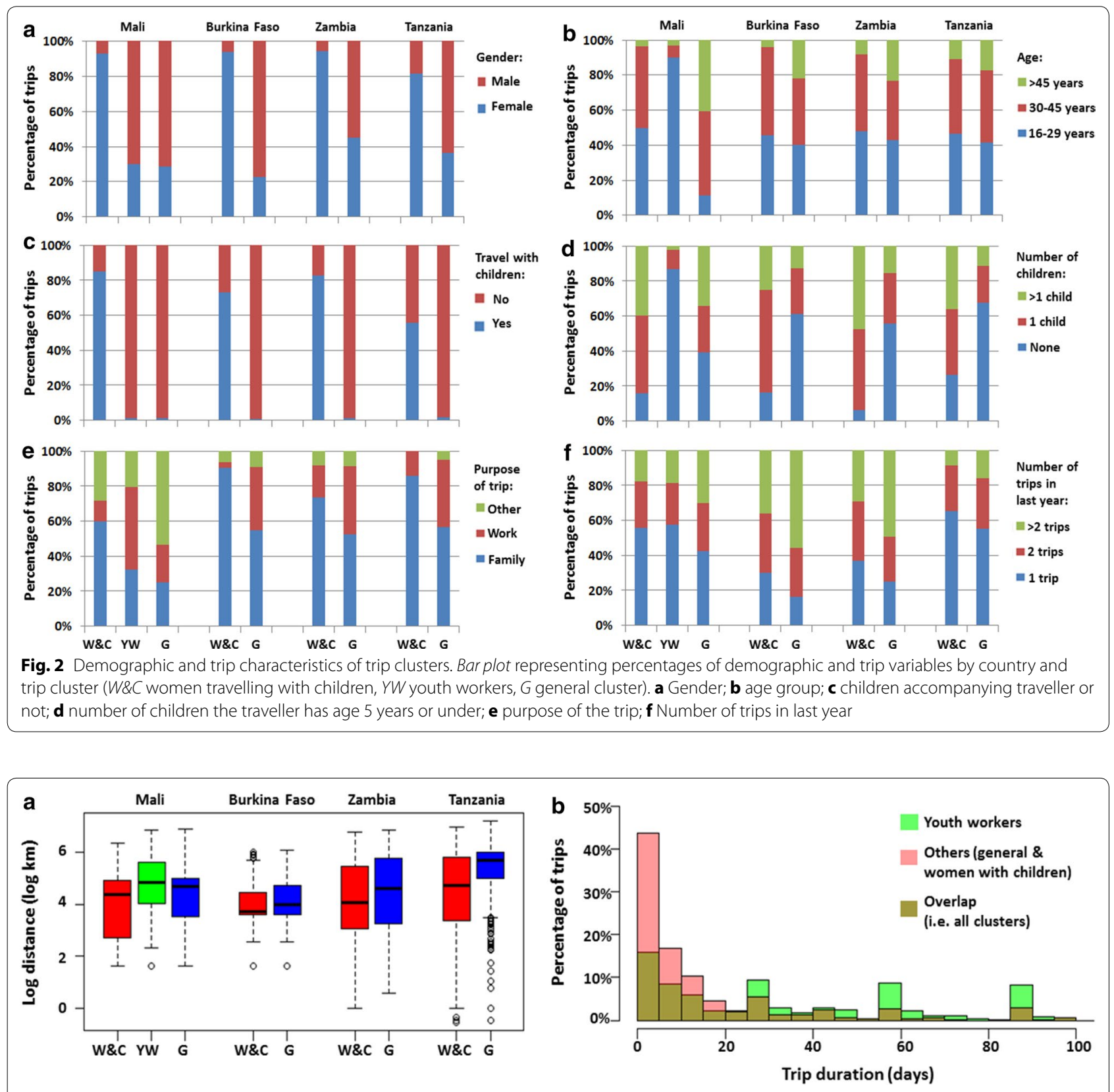

Fig. 3 Distance and duration distributions of trip clusters. a Box plot of log trip distances for the different trip clusters (W\&C women travelling with children, YW youth workers, G general cluster) by country. Median lines represent the 50th percentile and box edges represent the 25th and 75 th percentile of log trip distance. Circles represent outliers, and the lines outside the boxes represent the range of log trip distance excluding outliers. $\mathbf{b}$ Histogram of trip durations for youth workers and others (general and women travelling with children) for Mali

made by other groups. Travellers in the 'youth worker' cluster tended to make fewer trips than those in other trip clusters $(\mathrm{p}<0.001)$ - a mean of 1.7 trips in the last year compared to 2.2 trips for travellers in the 'women with children' cluster and 2.8 trips for 'general' travellers not belonging to these two clusters. Trips in the 'youth worker' cluster were also significantly longer in duration (mean of 70 days $c f 21$ days for trips in other clusters, $\mathrm{p}<0.001$ ) and involved larger distances (mean of $185 \mathrm{~km}$ cf $130 \mathrm{~km}$ for trips in other clusters, $\mathrm{p}<0.001)$. The vast majority of travellers in this group $(87 \%)$ had no children under the age of five.

The remaining trips belonged to a 'general' cluster. Travellers making trips in this 'general' cluster were predominantly male, belonged to all age groups, and trips in this cluster were for both family and work-related 
reasons (Fig. 2). In the Mali sample, many of the trips made by 16-29 year olds were absorbed by the 'youth worker' cluster and so travellers making trips in the 'general' cluster tended to be older than in the Burkina Faso, Zambia and Tanzania samples (mean of 44 years in the Mali sample cf 35 years in the samples from other countries, $\mathrm{p}<0.001)$ and to have more children under 5 years (mean of 1.11 children in the Mali sample $c f 0.56$ children in the samples from other countries, $\mathrm{p}<0.001$ ). Travellers making trips in the 'general' cluster tended to make more overnight trips per year than those making trips in the 'women with children' and 'youth worker' clusters (mean of 3.5 trips $c f 2.1$ trips for other clusters, $\mathrm{p}<0.001$ ). Trips in the 'general' cluster also tended to cover larger distances than those in the 'women with children' cluster (mean of $192 \mathrm{~km} c f 136 \mathrm{~km}$ for the 'women with children' cluster, $\mathrm{p}<0.001)$.

\section{Malaria risk factors}

Trips in the 'women with children' cluster were significantly more likely to be to areas of high malaria transmission than those in the 'general' cluster in the samples from Mali (OR $=4.46,95 \% \mathrm{CI}=3.42-5.83)$, Burkina Faso $(\mathrm{OR}=1.58,95 \% \mathrm{CI}=1.23-1.58)$, Zambia $(\mathrm{OR}=1.50$, $95 \% \mathrm{CI}=1.20-1.89$ ), and Tanzania (OR $=2.28,95 \%$ $\mathrm{CI}=1.71-3.05)$ (Table 2). Travellers making trips in the 'women with children' cluster were also significantly more likely to own a bed net in the samples from Burkina Faso $(\mathrm{OR}=1.77,95 \% \mathrm{CI}=1.25-2.53)$ and Zambia $(\mathrm{OR}=1.74,95 \% \mathrm{CI}=1.34-2.27)$. In the Zambia sample, travellers making trips in the 'women with children' sample had a significantly higher perceived risk of malaria at their destination than those making trips in the 'general' cluster $(\mathrm{OR}=1.35,95 \% \mathrm{CI}=1.03-1.77)$. In the Mali sample, trips in the 'youth worker' sample were significantly more likely to be to areas of high malaria transmission than those in the 'general' cluster $(\mathrm{OR}=23,95 \%$ $\mathrm{CI}=17-31)$.

\section{Mobile phone ownership and usage}

Travellers making trips in the 'women with children' cluster were significantly less likely to own a mobile phone than those making trips in the 'general' cluster in the samples from Mali $(\mathrm{OR}=0.50,95 \% \mathrm{CI}=0.39-0.65)$, Burkina Faso $(\mathrm{OR}=0.39,95 \% \mathrm{CI}=0.30-0.52)$ and Zambia (OR $=0.60,95 \% \mathrm{CI}=0.47-0.76)$ (Table 2). Of those who owned a mobile phone, travellers making trips in the 'women with children' cluster were significantly less likely to use their phone more than once per day compared to those making trips in the 'general' cluster in the samples from Mali $(\mathrm{OR}=0.67,95 \% \mathrm{CI}=0.46-0.96)$, Burkina Faso $(\mathrm{OR}=0.49,95 \% \mathrm{CI}=0.36-0.67)$ and Zambia (OR $=0.60,95 \% \mathrm{CI}=0.47-0.76$ ). Furthermore, in the Burkina Faso sample, trips in the 'women with children' cluster were significantly less likely to involve people travelling with phones compared to trips in the 'general' cluster $(\mathrm{OR}=0.35,95 \% \mathrm{CI}=0.18-0.68)$. Mobile phone sharing was relatively infrequent in all cases (less than $10 \%$ of interviewees in all four country samples reported sharing their phone with others). In the Mali sample, travellers making trips in the 'youth worker' cluster showed no significant difference in mobile phone ownership compared to travellers making trips in the 'general' cluster; but were significantly more likely to use their phone more than once per day $(\mathrm{OR}=1.37,95 \%$ $\mathrm{CI}=1.03-1.83)$ and were significantly less likely to travel with their phone $(\mathrm{OR}=0.34,95 \% \mathrm{CI}=0.14-0.76)$.

\section{Cross-border movement}

The survey also captured cross-border movements that were not included in the within-country analysis (Table 3). Cross-border movements were recorded in all countries except for Tanzania and represented $4.8 \%$ of all trips in the Mali sample, $4.7 \%$ of all trips in the Burkina Faso sample and $3.2 \%$ of all trips in the Zambia sample. International trips were predominantly made by male travellers while national trips were made by a smaller proportion of male travellers in the Burkina Faso sample (67\% male $c f 51 \%$ male for national trips, $\mathrm{p}=0.011)$ and the Zambia sample ( $60 \%$ male $c f 38 \%$ male for national trips, $\mathrm{p}<0.001)$. In the Burkina Faso sample, international trips were more frequently made by travellers belonging to the $30-45$ years old age group ( $54 \%$ were $30-45$ years of $40 \%$ for national trips, $\mathrm{p}=0.010)$. International trips were more likely to be for work-related reasons than national trips in all country samples (52\% work-related $c f 29 \%$ for national trips, $\mathrm{p}<0.001$ ) and, in the Burkina Faso sample, although international trips were less likely to be made by travellers owning a bed net (67\% ownership cf $81 \%$ for national trips, $\mathrm{p}=0.007$ ), international trips were more likely to be made by travellers using a bed net on their trip ( $41 \%$ used bed net $c f 29 \%$ of national trips, $\mathrm{p}=0.022$ ). International trips were more likely to be made by travellers owing a mobile phone than national trips in all country samples (82 \% ownership of $63 \%$ ownership for national trips, $\mathrm{p}<0.001)$. There were no significant differences in terms of children accompanying travellers for international or national trips. International trips tended to involve longer durations than national trips in the Mali sample (mean of 64 days $c f 37$ days for national trips, $\mathrm{p}=0.001$ ) and the Burkina Faso sample (mean of 63 days $c f 12$ days for national trips, $\mathrm{p}<0.001$ ), however, they were relatively shorter in the Zambia sample (mean of 11 days $c f 20$ days for national trips, $p<0.001$ ), possibly because several of the Zambian survey sites were border towns. 


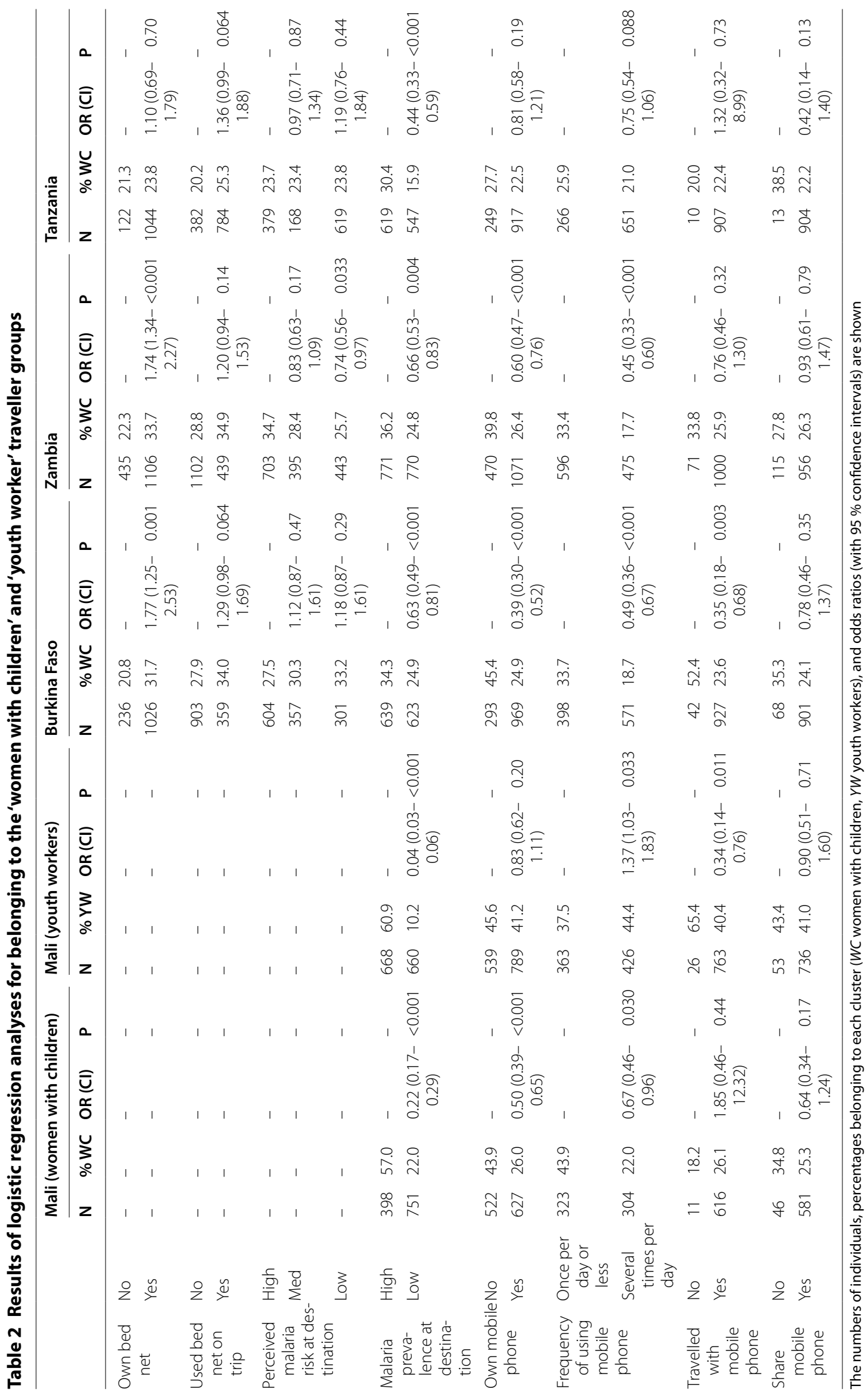


Table 3 Descriptive statistics for international travel from Mali, Burkina Faso and Zambia

\begin{tabular}{|c|c|c|c|c|c|c|}
\hline & \multicolumn{2}{|l|}{ Mali } & \multicolumn{2}{|c|}{ Burkina Faso } & \multicolumn{2}{|c|}{ Zambia } \\
\hline & $N$ & $\%(\mathrm{Cl})$ & $N$ & $\%(\mathrm{Cl})$ & $N$ & $\%(\mathrm{Cl})$ \\
\hline Total & 110 & & 76 & & 84 & \\
\hline \multicolumn{7}{|l|}{ Gender } \\
\hline Female & 41 & $37(28-47)$ & 25 & $33(23-45)$ & 34 & $40(30-52)$ \\
\hline Male & 69 & $63(53-72)$ & 51 & $67(55-77)$ & 50 & $60(48-70)$ \\
\hline \multicolumn{7}{|l|}{ Age } \\
\hline $16-29$ & 47 & $43(33-53)$ & 31 & $41(30-53)$ & 30 & $36(26-47)$ \\
\hline $30-45$ & 38 & $35(26-44)$ & 41 & $54(42-65)$ & 34 & $41(30-52)$ \\
\hline$>45$ & 25 & $23(15-32)$ & 4 & $5(1-13)$ & 19 & $23(14-33)$ \\
\hline Mean trip duration (range) & & $64.0(1-350)$ & & $62.8(1-365)$ & & $11.0(1-90)$ \\
\hline \multicolumn{7}{|l|}{ Purpose } \\
\hline Work & 47 & $43(33-53)$ & 49 & $64(53-75)$ & 43 & $51(40-62)$ \\
\hline Family & 46 & $42(32-52)$ & 24 & $32(21-43)$ & 29 & $35(24-46)$ \\
\hline Other & 15 & $14(8-21)$ & 2 & $2.6(0.3-9.2)$ & 11 & $13(7-22)$ \\
\hline \multicolumn{7}{|l|}{ Own bed net } \\
\hline Yes & N/A & N/A & 51 & $67(55-77)$ & 61 & $73(62-82)$ \\
\hline No & N/A & $\mathrm{N} / \mathrm{A}$ & 25 & $33(23-45)$ & 23 & $27(18-38)$ \\
\hline \multicolumn{7}{|l|}{ Use bed net on trip } \\
\hline Always & N/A & $\mathrm{N} / \mathrm{A}$ & 30 & $39(28-51)$ & 19 & $23(14-33)$ \\
\hline Sometimes & N/A & $\mathrm{N} / \mathrm{A}$ & 0 & $0(0.0-4.7)$ & 3 & $3.6(0.7-10.1$ \\
\hline Never & N/A & $\mathrm{N} / \mathrm{A}$ & 44 & $58(46-69)$ & 61 & $73(62-82)$ \\
\hline \multicolumn{7}{|l|}{ Own mobile phone } \\
\hline Yes & 80 & $73(63-81)$ & 69 & $91(82-96)$ & 70 & $83(74-91)$ \\
\hline No & 30 & $27(19-37)$ & 6 & $8(3-16)$ & 12 & $14(8-24)$ \\
\hline
\end{tabular}

The number of individuals $(N)$ and percentage of the sample (exact binomial $95 \%$ confidence interval), or mean (range) are shown. All interviewees did not answer all questions, hence there are some missing values

From the Mali survey sites, international travel was most commonly reported to other West African countries, such as Côte d'Ivoire, Guinea, Senegal, Burkina Faso, and Ghana, with occasional trips to France, the former colonial power, and Saudi Arabia for religious worship (Additional file 4: Figure S1), in agreement with the qualitative literature $[31,32]$. From the Burkina Faso survey sites, international travel was most commonly reported to other West African countries, such as Côte d'Ivoire, Ghana, Benin, Togo, Mali, Senegal, and Niger. From the Zambia survey sites, international travel was most common to other East and Southern African countries, such as Malawi, Tanzania, South Africa, Namibia, Botswana, and the Democratic Republic of Congo. However, it should be noted that these destinations likely depend on the geographical distribution of the survey sites. Nevertheless, they reflect a high degree of informal cross-border movement, much of which is unlikely to be captured in international statistics on either migration or in air travel data.

\section{Discussion}

Data on human movement patterns were collected and analysed in four countries in West, East and Southern Africa. The West African countries-Mali and Burkina Faso-are of interest because they are affected by seasonal agricultural labour movements [5, 15], while the Southern/East African countries-Zambia and Tanzania-have more comprehensive national transport networks [24] and youth movements due to boarding school [20]. Interestingly, despite differences in culture and geography, a cluster of trips was observed representing a group of travellers-women with children-that displayed very similar travel patterns across all four countries. In addition, a cluster of trips was observed representing a second major traveller group-youth workers-in the Mali data set, which corresponds very well with the qualitative literature [5, 15-17]. This is the first time that the movement patterns of these traveller groups have been quantified. 
The 'women with children' trip cluster consists predominantly of trips made by women between the ages of 16 and 45 years who travel with children, usually for family-related reasons. These travellers tend to make fewer trips than other travellers and tend to travel shorter distances. In the samples from Mali, Burkina Faso, Zambia, and Tanzania, they were significantly more likely to travel to areas of high malaria prevalence, although in the Zambia sample, they had a higher perceived risk of malaria. In the Burkina Faso and Zambia samples, they were more likely to carry out malaria prevention measures, such as owning a bed net. Their travel to high prevalence areas is relevant to malaria transmission because the children that accompany them are more susceptible to malaria infection and may carry parasites with them back to the origin of travel. Considering the children that accompany them, this traveller group is expected to contribute greatly towards spatial malaria transmission. Additionally, some women who travel with children may also be pregnant, causing them to be more susceptible to malaria infection themselves, thus enhancing their contribution to spatial malaria transmission.

The Malian 'youth worker' trip cluster consists predominantly of trips made by young men and a significant number of women between the ages of 16 and 29 years who travel without children, usually for work-related reasons, on trips that last on the order of months. The lower age limit for this group corresponds to our eligibility criteria, and hence it is possible that travellers slightly younger than 16 years may also display similar travel patterns. These travellers tend to make fewer trips per year but their trips tend to involve longer distances compared to other traveller groups. They are also significantly more likely to travel to areas of high malaria prevalence, increasing their risk of contracting malaria and carrying it back to their origin of travel. The longer duration of their trips also provides more time for malaria transmission. It is of interest that this traveller group emerged from the analysis because it corresponds to a traveller group previously described only by qualitative research $[5,15-17]$. It is expected that youth workers make a significant contribution to spatial malaria transmission in Mali because their movements correlate with seasonal rains and hence peak mosquito densities. The seasonality to these movements was not captured by the cluster analysis, possibly due to trip departure dates not corresponding with the seasonal definitions, however qualitative studies highlight the seasonal dimension of youth worker movements, as agricultural labour is highest during the rainy season when malaria is also most prevalent $[5,33]$, further highlighting the importance of this group to spatial malaria transmission in the Sahel.
With anonymous mobile phone signal data increasingly being used as a proxy for human movement patterns $[2,11]$, results from this survey on mobile phone usage behaviour can help quantify the biases inherent in this data source. In the samples from Mali, Burkina Faso and Zambia, travellers making trips in the 'women with children' cluster were significantly less likely to own a mobile phone than other travellers, and if they did own one, were significantly less likely to use it more than once per day. In the Burkina Faso sample, travellers making trips in the 'women with children' cluster were significantly less likely to travel with their phones compared to other travellers, but this trend was not seen in the other country samples. Phone sharing was relatively infrequent in all country samples (less than $10 \%$ of interviewees claimed to share their phone), and no significant differences were seen between travellers making trips in the 'women with children' cluster and other travellers in terms of phone sharing, in contrast to previous results that rural women tend to share their phones more frequently [13]. Travellers making trips in the 'youth worker' cluster in Mali showed no significant difference in mobile phone ownership compared to travellers making trips in the 'general' cluster, but were significantly more likely to use their phones more than once per day and, surprisingly, were significantly less likely to travel with their phones. These results show that mobile phone usage behaviour is country-specific and hence mobile phone signal data may require country-specific corrections.

Several weaknesses of this study should be acknowledged when interpreting the results. The survey sites (Fig. 1) were a judgement/convenience sample chosen to: (a) capture a wide range of traveller groups; and, (b) take advantage of existing relationships with the local researchers. Since the study sites were not chosen randomly, inferences based on these results can not be assumed to be representative of each country as a whole, and instead represent the collection of study sites surveyed. Different locational biases were present in each country-in Tanzania, most of the survey sites were relatively urban, and in Burkina Faso, the survey sites were in or within the vicinity of the capital city, Ouagadougou. In Zambia, several of the survey sites were border towns, possibly explaining the short duration cross-border trips, and in Mali, the survey sites were relatively rural, which could partly explain the lower level of mobile phone ownership recorded there. The results analysed here provide a snapshot of traveller groups in a sub-set of each country, and could form a basis for larger-scale studies randomized at a national level. Cluster sampling could be implemented here by applying the principle of probability sampling to each national administrative level sequentially [34]. 
Other shortcomings include biases in the recording of trips and social desirability bias. Regarding trip recording, interviewees were asked in the questionnaire about their three most recent short-term and long-term trips (up to six in total). This could introduce a bias towards trips in the months preceding the interviews in two ways-recall bias, since recent trips may be easier to remember; and 'trip clipping' since, for people who have taken many trips, only the recent ones will be recorded. Furthermore, certain questions are subject to social desirability bias, since respondents may feel inclined to give the 'right' answer (e.g., questions on bed net and phone ownership and usage) [35].

\section{Conclusions}

As a growing number of diseases are targeted for elimination, including poliomyelitis, Chagas disease and neglected tropical diseases, such as schistosomiasis, lymphatic filariasis and onchocerciasis, the role of travellers in sustaining transmission will become increasingly important. The combined survey and cluster analysis approach outlined here provides a powerful framework for identifying key traveller groups that, in combination with knowledge of the local epidemiology of disease transmission, will help to inform disease elimination programmes. Information on key traveller groups will also help to inform control programmes for emerging pathogens, such as Ebola [36], Zika [37], and pathogens yet to emerge [38].

A study following this approach randomized at a national scale is needed to infer nationally representative traveller groups, however, the survey results analysed here provide a snapshot of traveller groups present in a collection of sites in Mali, Burkina Faso, Zambia, and Tanzania. These provide preliminary evidence for the generality of the 'women with children' traveller group across all four countries, despite significant differences in culture and geography. Results from the Mali survey also provide preliminary evidence for youth workers as a key traveller group, in agreement with previous qualitative studies [5, 15-17].

Further survey work is encouraged, and questions recommended for inclusion in future DHS and Malaria Indicator Surveys to better characterise traveller groups are included in Additional file 1. However, given the expected contributions of these two groups to spatial malaria transmission-women with children due to the children they travel with having high parasite prevalence, and youth workers due to their movements being correlated with seasonal rains and peak mosquito densities-it is recommended that interventions aimed at interrupting spatial malaria transmission consider these groups. A number of interventions are available or being considered to address malaria importation including enhanced surveillance, targeted mass drug administration and provision of bed nets to travellers. Further studies utilizing field work, data analysis and mathematical models will help to develop a deeper understanding of the likely impact of these measures and to design optimal delivery strategies in a wide range of settings.

\section{Availability of supporting data}

The data sets supporting the results of this article are available in Additional file 2.

\section{Additional files}

Additional file 1. Survey protocol, data and analysis. Details of the workflow followed in each survey village/community, the sampling technique and data processing. Impact of survey timing on results. Questions recommended for inclusion in future Demographic and Health Surveys and Malaria Indicator Surveys to better characterise traveller groups.

Additional file 2. Demographic and trip data for each survey country. Excel file containing two data sheets for each survey country-a traveller data sheet with a separate row for each interviewee and demographic variables as columns, and a trip data sheet with a separate row for each recorded trip and trip variables as columns. Trips are indexed with a unique TripID are matched to interviewees via a unique PersonID. Demographic and trip variables are summarized in "Traveller Variables" and "Trip Variables" sheets of the Excel file, respectively.

Additional file 3: Table S1. Descriptive statistics and trip clusters for the two Mali surveys.

Additional file 4: Figure S1. International destinations recorded in surveys in Mali, Burkina Faso and Zambia.

\section{Abbreviations}

DHS: Demographic and Health Survey; OR: odds ratio.

\section{Authors' contributions}

JMM, ACG, TDH, and NMF designed the research project. MBT, SD and JMM conducted the survey in Mali. ALO, AR and JMM conducted the survey in Burkina Faso. MN, EN and JMM conducted the survey in Zambia. SSK, NJG and JMM conducted the survey in Tanzania. JMM, ACG and NMF analysed the data. JMM wrote the first draft of the manuscript. All authors edited the manuscript. All authors read and approved the final manuscript.

\section{Author details}

${ }^{1}$ Department of Infectious Disease Epidemiology, MRC Center for Outbreak Analysis and Modelling, Imperial College London, London, UK. ${ }^{2}$ Divisions of Biostatistics and Epidemiology, School of Public Health, University of California, Berkeley, CA, USA. ${ }^{3}$ Malaria Research and Training Center, University of Bamako, Bamako, Mali. ${ }^{4}$ Centre National de Recherche et de Formation sur le Paludisme, Ouagadougou, Burkina Faso. ${ }^{5}$ Institute for Disease Modeling, Bellevue, WA, USA. ${ }^{6}$ Chainama College of Health Sciences, Lusaka, Zambia. ${ }^{7}$ Environmental Health and Ecological Sciences Thematic Group, Ifakara Health Institute, Dar es Salaam, Tanzania. ${ }^{8}$ School of Life Sciences, University of Warwick, Warwick, Coventry, UK.

\section{Acknowledgements}

We thank Dr. David Aanensen, Dr. Derek Huntley and Chris Powell for assistance with the EpiCollect 2.0 interface. We also thank the survey teams in each of the four countries surveyed-Dr. Nina Madjako Soumahoro-Toure, Dr. Moctar Kardigue Coulibaly, Dr. Kadiatou Kone, Dr. Mohamed Serge Toure, Siaka Traore, and Gaoussou Sogoba in Mali; Apollinaire Nombre, Malik Lankoande, Kadija Ouedraogo, Lassena Kabore, Moussa Rabo, Marcel Ouedraogo, Houd Kanazoe, and Anassa Thiombiano in Burkina Faso; Maureen Mwambazi, 
Audrey Mulungushi, Joseph Daka, Gabriel Karumia, Samuel Daka, and Kanyatta Kanyatta in Zambia; and Sambo Maganga, Daud Mbwana, Patrick Nshana, John Lyatuu, Happyness Kasomangala, and Jane Masamu in Tanzania. We additionally thank Prof Chris Drakeley and Dr. Teun Bousema for input on survey design. This research was jointly funded by the UK Medical Research Council (MRC) and the UK Department for International Development (DFID) under the MRC/DFID Concordat agreement. JMM acknowledges additional support from a fellowship from the MRC/DFID.

\section{Competing interests}

The authors declare that they have no competing interests.

Received: 7 January 2016 Accepted: 30 March 2016 Published online: 12 April 2016

\section{References}

1. Lynch C, Roper C. The transit phase of migration: circulation of malaria and its multidrug-resistant forms in Africa. PLoS Med. 2011;8:e1001040.

2. Wesolowski A, Eagle N, Tatem AJ, Smith DL, Noor AM, Snow RW, et al. Quantifying the impact of human mobility on malaria. Science. 2012;338:267-70.

3. WHO. World malaria report 2013. Geneva: World Health Organization; 2013.

4. O'Meara WP, Mangeni JN, Steketee R, Greenwood B. Changes in the burden of malaria in sub-Saharan Africa. Lancet Infect Dis. 2010;10:545-55.

5. Prothero RM. Migration and malaria risk. Health Risk Soc. 2001;3:19-38.

6. Bousema T, Griffin JT, Sauerwein RW, Smith DL, Churcher TS, Takken W, et al. Hitting hotspots: spatial targeting of malaria for control and elimination. PLoS Med. 2012;9:e1001165.

7. Sturrock HJ, Hsiang MS, Cohen JM, Smith DL, Greenhouse B, Bousema $\mathrm{T}$, et al. Targeting asymptomatic malaria infections: active surveillance in control and elimination. PLoS Med. 2013;10:e1001467.

8. Dondorp AM, Fairhurst RM, Slutsker L, MacArthur JR, Breman JG, Guerin PJ, et al. The threat of artemisinin-resistant malaria. N Engl J Med. 2011;365:1073-5.

9. Pindolia DK, Garcia AJ, Wesolowski A, Smith DL, Buckee CO, Noor AM, et al. Human movement data for malaria control and elimination strategic planning. Malar J. 2012;11:205.

10. Tatem AJ, Huang ZJ, Narib C, Kumar U, Kandula D, Pindolia DK, et al. Integrating rapid risk mapping and mobile phone call record data for strategic malaria elimination planning. Malaria J. 2014;13:52.

11. Tatem AJ, Qiu YL, Smith DL, Sabot O, Ali AS, Moonen B. The use of mobile phone data for the estimation of the travel patterns and imported Plasmodium falciparum rates among Zanzibar residents. Malar J. 2009;8:287.

12. Communications Regulatory Authority of Namibia. Universal Service Baseline Study. Windhoek: Government of Namibia; 2011.

13. Wesolowski A, Eagle N, Noor AM, Snow RW, Buckee CO. The impact of biases in mobile phone ownership on estimates of human mobility. J Roy Soc Interface. 2013;10:20120986.

14. Tanzania Demographic and Health Survey. Macro International. 2010.

15. Prothero RM. Populations on the move. Third World Q. 1987;9:1282-310.
16. Remy G. Mobilité géographique et immobilisme social: un exemple voltaique. Revue Tiers Monde. 1977;18:617-53.

17. Amselle J. Migration et société néotraditionelle: Le cas des Bambara du Jitumu (Mali). Cahiers d'Etudes Africaines. 1978;18:487-502.

18. Findley SE. Does drought increase migration - a study of migration from rural mali during the 1983-1985 drought. Int Migr Rev. 1994;28:539-53.

19. Dougnon I. Migratory trends among two Malian ethnic groups, the Songhai and the Dogon, migrating to Ghana: a comparative study. Bamako: University of Bamako; 2010.

20. Gould WTS. Circulation and schooling in East Africa. In: Prothero RMCM, editor. Circulation in Third World Countries. London: Routledge; 1985. p. 262-78.

21. Donnelly MJ, McCall PJ, Lengeler C, Bates I, D'Alessandro U, Barnish G, et al. Malaria and urbanization in sub-Saharan Africa. Malar J. 2005;4:12.

22. Parnell S, Walawege R. Sub-Saharan African urbanisation and global environmental change. Global Environ Chang. 2011;21:S12-20.

23. Dougnon I. Migration and small scale irrigation in a post-conflict region of Mali. Bamako: University of Bamako; 2006.

24. The World Factbook. Central Intelligence Agency. 2015.

25. Aanensen DM, Huntley DM, Feil EJ, al-Own F, Spratt BG. EpiCollect: linking smartphones to web applications for epidemiology, ecology and community data collection. PloS One. 2009;4:e6968.

26. Le S, Josse J, Husson F. FactoMineR: an R package for multivariate analysis. J Stat Softw. 2008;25:1-18.

27. Gething PW, Patil AP, Smith DL, Guerra CA, Elyazar IRF, Johnston GL, et al. A new world malaria map: plasmodium falciparum endemicity in 2010. Malar J. 2011;10:378.

28. Mali Demographic and Health Survey. Macro International. 2006.

29. Zambia Demographic and Health Survey. Macro International. 2007.

30. Burkina Faso Demographic and Health Survey. Macro International. 2010.

31. Le Adams A. Long Voyage des Gens du Flueve. Paris: Francois Maspero; 1977.

32. Coulibaly S, Gregory J, Piche V. Les Migrations Voltaiques: importance et ambivalence de la Migration Voltaique. Ottawa: Centre Voltaique de la Recherche Scientifique, Institut National de la Demographie; 1980.

33. Saxena VK, Devadethan MA. Impact of the seasonal migration of labour forces on the spread of malaria. Ann Trop Med Parasitol. 1998;92:821-2.

34. Henry GT. Practical Sampling. London: Sage; 1990.

35. Oppenheim AN. Questionnaire design, interviewing and attitude measurement. London: Pinter Publishers; 1992.

36. WHO Ebola Response Team. Ebola virus disease in West Africa-the first 9 months of the epidemic and forward projections. New Engl J Med. 2014;371:1481-95.

37. Bogoch II, Brady OJ, Kraemer MUG, German M, Creatore MI, Kulkarni MA, et al. Anticipating the international spread of Zika virus from Brazil. Lancet. 2016;335:335-6.

38. Morens DM, Fauci AS. Emerging infectious diseases: threats to human health and global stability. PLoS Pathog. 2013;9:e1003467.

\section{Submit your next manuscript to BioMed Central and we will help you at every step:}

- We accept pre-submission inquiries

- Our selector tool helps you to find the most relevant journal

- We provide round the clock customer support

- Convenient online submission

- Thorough peer review

- Inclusion in PubMed and all major indexing services

- Maximum visibility for your research

Submit your manuscript at www.biomedcentral.com/submit

C Biomed Central 